\title{
Case-Based Reasoning for Adaptive Aluminum Extrusion Die Design Together with Parameters by Neural Networks
}

\author{
S. Butdee and S. Tichkiewitch
}

\begin{abstract}
Nowadays Aluminum extrusion die design is a critical task for improving productivity which involves with quality, time and cost. Case-Based Reasoning (CBR) method has been successfully applied to support the die design process in order to design a new die by tackling previous problems together with their solutions to match with a new similar problem. Such solutions are selected and modified to solve the present problem. However, the applications of the CBR are useful only retrieving previous features whereas the critical parameters are missing. In additions, the experience learning to such parameters are limited. This chapter proposes Artificial Neural Network (ANN) to associate the $\mathrm{CBR}$ in order to learning previous parameters and predict to the new die design according to the primitive die modification. The most satisfactory is to accommodate the optimal parameters of extrusion processes.
\end{abstract}

Keywords Adaptive die design and parameters - Optimal aluminum extrusion - Case-based reasoning - Neural networks

\section{Introduction}

Aluminum extrusion is a hot deformation process used to produce long, straight, semi finished metal products such as bars, solid and hollow sections, tubes and many shapes of products. The hot aluminum extrusion process is pressed under high pressure and temperature in a specific machine. A

\footnotetext{
S. Butdee

Department of Production Engineering, Faculty of Engineering, King Mongkut's University of Technology North Bangkok, Bangkok 10800,

Thailand

e-mail: stb@kmutnb.ac.th

S. Tichkiewitch

G-SCOP Laboratory, Grenoble Institute of Technology, Grenoble, France
}

billet is squeezed from close container to be pushed through a die to reduce its section [1]. A profile shape is deformed in order to be adapted to the die orifice shape on an aluminum extrusion press. The main tooling of aluminum extrusion process is a die which is performed aluminum profile shapes. The die design is a critical task for improving productivity which involves with quality, time and cost. Case-Based Reasoning (CBR) method has been successfully applied to support the die design process in order to design a new die by tackling previous problems together with their solutions to match with a new similar problem. Such solutions are selected and modified to solve the present problem. However, the applications of the CBR are useful only retrieving previous features whereas the critical parameters are missing. In additions, the experience learning to such parameters are limited. This chapter proposes Artificial Neural Network (ANN) to associate the CBR in order to learning previous parameters and predict to the new die design according to the primitive die modification. Artificial neural network methodology applied to solve the complex or specific problem in engineering works [2]. The case study of an example die design is discussed.

\section{Aluminium Extrusion Die Design}

Die design is one of crucial task in aluminum extrusion process. The success and failure cases are studied and analyzed in order to use their information from these cases for improving a new die design to avoid failure case. In practice, a die may be tested many times before the extrusion profile is satisfactory. If the extruded profile shape is not perfect, die geometry will be modified such as bearing length to balance extrusion speed of the section for obtaining good quality profile shape. The quality factors include a less number of testing, long life of the die, high productivity and so on. In general, die design process consists of selection of a die type and size, calculation profile section shrinkage, determination hot aluminum flow controlling, determination 
of die orifice dimension and deflection of tongue, calculation of the bearing lengths, supporting tool design. Each die design process should be focused on the parameters that have effect to die efficiency such as shape factor, extrude ability, extrusion ratio, etc. In fact, die design or die geometry will be modified following the profile characteristics, especially, profile shapes and dimensions. In term of efficient operators, they should have a thorough understanding of the different functions of each die features (i.e. leg, sink in, porthole, bearing etc.). These features will be modified to interact with the process parameters and each of the product characteristics. The complex flow of material in the extrusion die or container often creates different deformation conditions in various regions of an extruded product. Die design has to imagine the metal flow pattern in die and container in order to make decisions for the definition of each die feature. Especially, die orifice and bearing length are the important features, which have directly affected with metal flow velocity. In theory, flow velocity of each point on the section should be balanced to avoid the extruded profile twist.

The die design is based on skills and experiences from a die designer, who accumulates the knowledge of die design for using this knowledge to solve the die design problems. Decision making for designing he die consists of selection of the suitable press, selection of type of die and its materials, laying out the profile(s) on the die in order to optimize the extrusion yield, handling with unbalance velocity of the metal flow on each point of the sections, the consolidation of the tooling to withstand in extrusion process, etc. CAD is used to associate a designer to improve productivity not only be able to reduce time but also can ensure a quality of die design. Feature based technique is one of the most popular used to support the CBR method in the phase of rapid modification.

\section{Feature Based Library}

Traditionally, CAD tooling offered for three methods; Solid modeling, Wire-frame modeling and Surface modeling. Solid modeling is one of the effective modeling which is the most similar object to the real part [3]. However, the solid model is still needed to develop in the aspects of fast design respond and design based on the previous parts or features. Therefore, the feature based technology was emerged [4]. The principles of feature can be several characteristics. They are form features, function features, tolerance features, assembly features, material features and property features. The original concept of feature base is derived variant approach of process planning which is believed that the similar parts are should have the similar process plans. The variant approach contains four steps. First is collected the existing process plans and stored in the library. Second, the stored plans are classified, coded and indicated. Third, the new part is input and matched to the system library with the same or similar parts. The matched parts are retrieved and shown. The information is shown which are not only the parts, but also their process plans. Fourth, one or many of the shown process plans are selected and modified to fit with the new part. This concept can save a lot of time and can be applied to many fields of industrial manufacturing; design, planning, production, etc. Feature-based design tools have been applied to many applications. Thomson [5] stated the applications of the feature concept to reverse engineering to machine maintenance. The paper explained that the method of using CMM in order to get machine part features and then recorded them in the library. Gayreti and Abdalla [6] expressed a feature based prototype system for the evaluation and optimization of manufacturing processes. A feature is defined as generic shape carrying product information, which aid design or communication between design and manufacturing.

Die geometry can be classified into three categories. They are solid, hollow and semi-hollow die. The difference die geometries provide the various shapes of the end product profiles. Figure 1 shows the example of feature based extrusion die which is recorded in the case library. The die assembly (AC245/1) composes of three components. They are feeder plate (FPAC245-01), die plate (DPAC245-01), and back plate (BKAC245-01). Each component has their own feature class, whereas some of them shares with the common class. For example, the open pocket, The close pocket, the edge chamfer feature class can share with the three components. By constant, the tap hole is specifically used for the feeder plate feature class. The class benefits to manage on the process planning method selection. The feature based die library

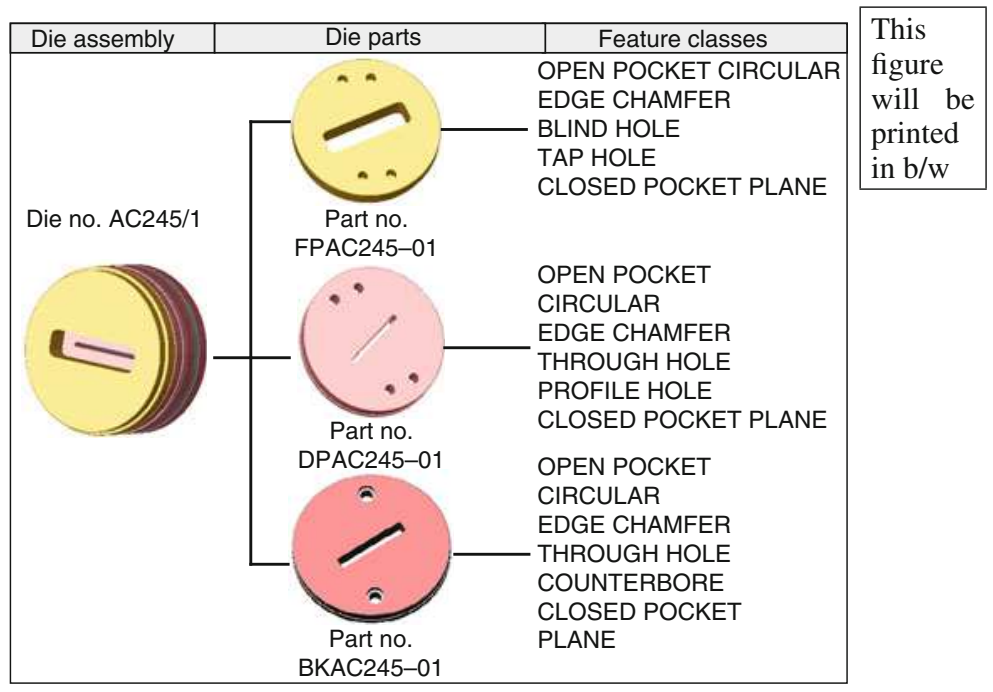

Fig. 1 Example of feature based extrusion die 
has advantages when they are stored with a well-structured approach. The main component is the die plate, whereas the back plate and feeder plate can be shared with different die plate in the same class. The case library is normally organized together with case-based reasoning which is explained in the next section.

\section{Case-Based Reasoning Method}

The concept of case based reasoning can be defined in the way to organize information or data, and this concept is applied to either 'idea', innovation or any other kinds of information that is to be stored and used in the future. Case-based reasoning (CBR) has applied to several domains. Design is one of the areas which is used in order to reduce design lead time together with to capture knowledge from experts and reuse such knowledge when the experts are not available. The basic idea of case-based reasoning is that new problems can be tackled by adapting solutions that were used to solve previous problems [7]. It is shown by many papers that are more efficient to solve problems by using previous similar solutions than to generate the entire solutions from scratch. The example applications are in the areas of architecture design [8], in chemical process engineering [9], and in mechanical design [10] as well as design for mass customization [11] etc. CBR consists of four main elements; case problem identification, case retrieval, case adaptation, case storage. All of the cases are linked to data case library that is accepted as knowledge-based library. In addition, there are another two components that assist the case retrieval and the case adaptation. They are case indexing method and case modification rule respectively.

Figure 2 shows the cycle of case-based reasoning. Primitive cases of die features are collected and stored in the

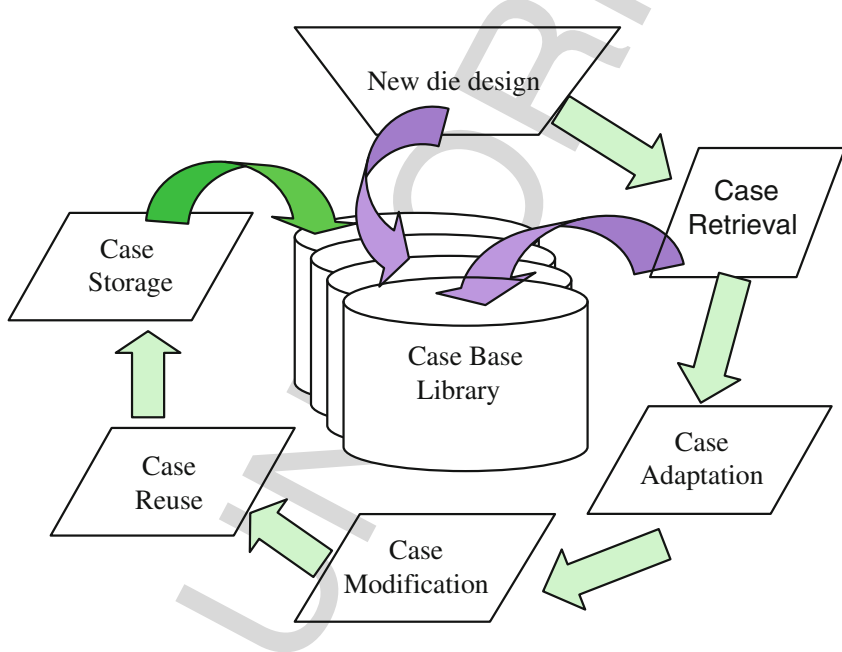

Fig. 2 Cycle of case-based reasoning (CBR)

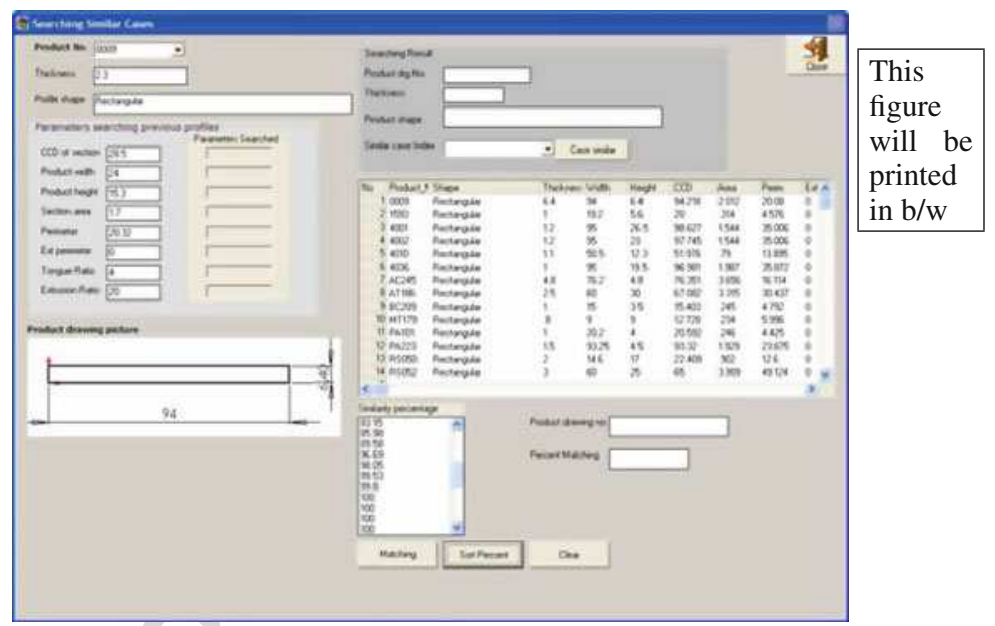

Fig. 3 Feature comparison and matching

case library. Such die features are classified and indexes in order to simply retrieve whenever it needed. New die design attributes are identified and put into the CBR cycle. The case retrieval is matched and retrieved the same or similar die features. If the same die is fortunately found, it will be adapted and used for the new die design. If it is not, the similar cases are taken into account. The most similar die is selected and adapted. It is then modified by an expert designer. The modified die is tested and reused for the new order in the extrusion process. The successful and failure are observed as well as recorded inside the case library for the future use. Figure 3 shows the case-based reasoning program which has been developed by Visual Basic which links to a MS access database and CAD (Solidworks ${ }^{\odot}$ ). The new aluminum profile is drawn on the CAD and export to the CBR program with it attributes. They are thickness, width, height, CCD, section-area, perimeter, tongue ratio, and extrusion ratio.

The program output shows the matching result in the degree of percentages. The most similar case or the most desire is selected. This program is able to bring the user to access to the CAD automatically in order to do a modification. Although, the CBR method is helpful, it can associate on the symbolic pattern matching. The significant numerical parameters are loosed. Therefore, the ANN method is considered to corporate.

\section{Adaptive Parameters With Artificial Neural Networks}

Artificial neural network is a mathematical model for parallel computing mechanisms as same as biological brain. They consist of nodes, linked by weighted connections. Neural networks are constructed by hierarchical layers, which are 
Fig. 4 Hierarchy of neural network procedure

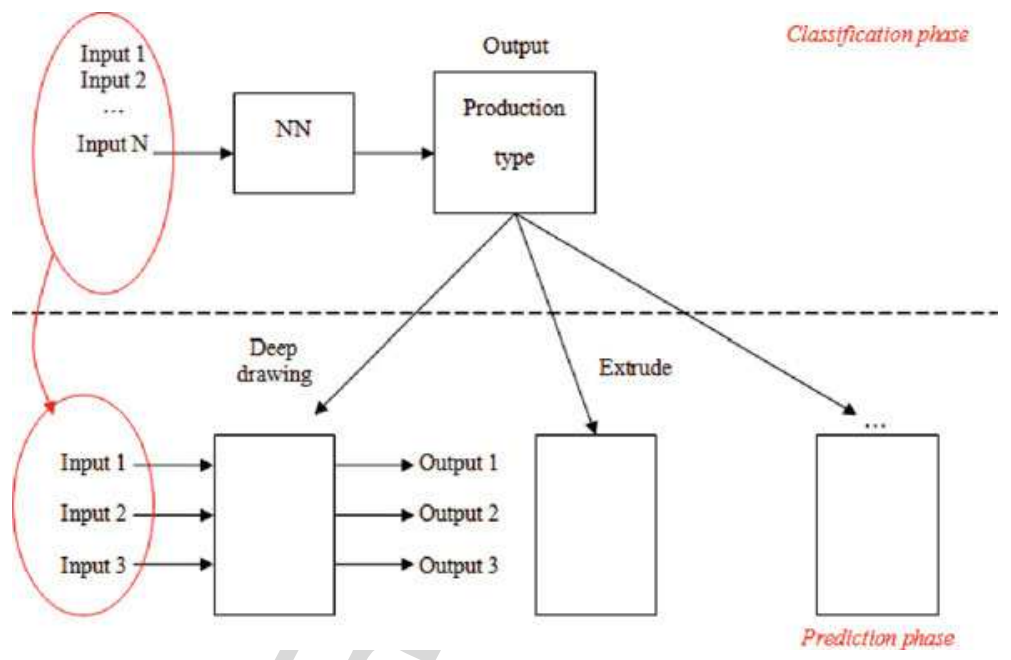

input, hidden, and output layer respectively. Neural networks learn relationships between input and output by iteratively changing interconnecting weight values until the outputs over the problem domain represent the desired relationship. Praszkiewicz [12] purposed the application of neural network to the determination in small lot production in machining. A set of features considered as input vector and time consumption in manufacturing process was presented and treated as output of the neural net. Scholz et al. [13] presented casebased reasoning for production control with neural networks, whereby expert knowledge about neural production control could be processed and structured in such a way that control strategies and according to, the applicable and adjusted neural networks could be selected and implemented for new production situations. The case-based reasoning enables the output of a solution for existing cases or the adding of new cases of neural production control.

Figure 4 shows the hierarchy of neural network procedure when it is applied to assist the CBR. There is divided into two steps. The first step is performed for classification, whereas the second step is used for prediction. The first result is considered together with the new data input. The classical concept of ANN is explained as the followings.

The mathematical model of the biological neuron, there are three basic components as presented in Fig. 5. First, the synapses of the neuron are modeled as weights. The value of weight can be presented the strength o the connection between an input and a neuron. Negative weight values reflect inhibitory connections, while positive values designate excitatory connections. Second component is the actual activity within the neuron cell. This activity is referred to as linear combination. Finally, an activation function controls the amplitude of the output of the neuron. An acceptable range of output is usually between 0 and 1 , or -1 and 1 .

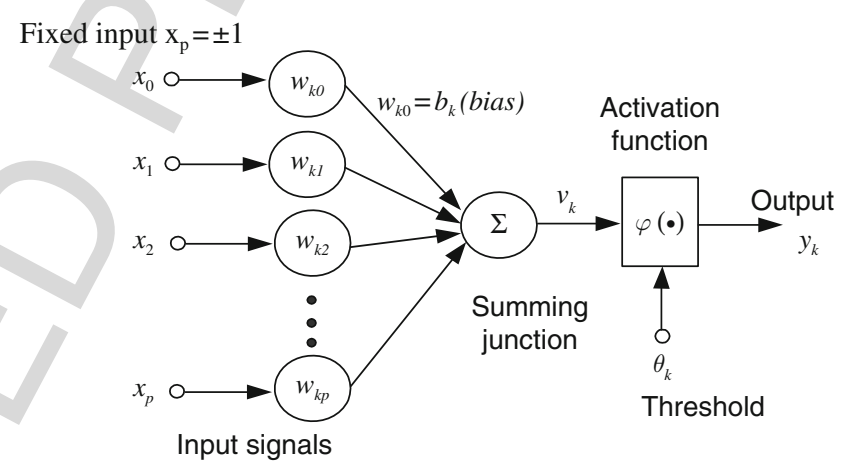

Fig. 5 A perceptron neuron model

Each neuron calculates three functions. The first is propagation function as shown in Eq. (1),

$$
v_{k}=\sum w_{k j} x_{j}+b_{k}
$$

where $w_{k j}$ is the weight of the connection between neuron $k$ and $j, y_{k}$ is the output from neuron $k$, and $b_{k}$ is the bias. The second is an activation function. The output of a neuron in a neural network is between certain values (usually 0 and 1 , or -1 and 1). In general, there are three types of activation functions, denoted by $\phi(\bullet)$. Firstly, there is the treshold function which takes on a value of 0 if the summed input is less than a certain threshold value (v), and the value 1 if the summed input is greater than or equal to the threshold value.

$$
\varphi(v)=\left\{\begin{array}{l}
1 \text { if } v \geq 0 \\
0 \text { if } v<0
\end{array}\right.
$$

The proposed structure of neural network for die design and process planning is shown in Fig. 6. The input 
213

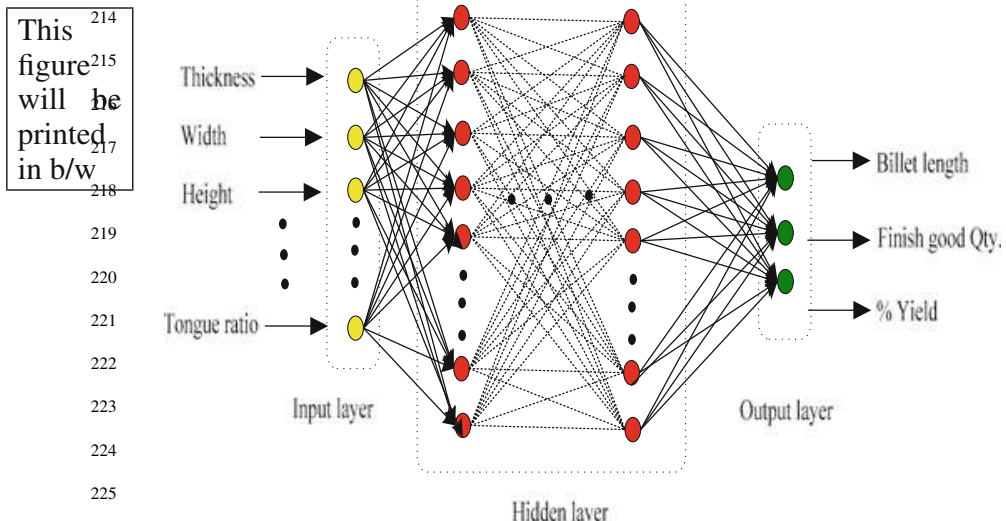

Fig. 6 The structure of neural networks

parameters consist of the characteristics of aluminium profile including type of profile, profile shape, dimensions, cross-section area, extrusion ratio, CCD (Circumscribing Circle Diameter), tongue ratio etc. The output layer contains billet length, profile finished length and degree of yield.

The neural network has been trained by using the standard which is one of three categories of the training method. Supervised learning may be called associative learning, is trained by providing with input and matching output patterns. These input-output pairs can be contributed by an external teacher, or by the system which contains the neural networks (self-supervised). The learning process or knowledge acquisition takes place by representing the network

Table 2 The output parameters
Fig. 7 The training and learning process
Table 1 The sample test input parameters

\begin{tabular}{ll}
\hline Item & Value \\
\hline Thickness & 2.3 \\
Width & 24 \\
Height & 15.3 \\
CCD & 28.5 \\
Area & 1.7 \\
Perim & 20.32 \\
Tounge ratio & 4 \\
ER & 20 \\
Number of die hole & 1 \\
Extrusion ratio via number of die hole & 20 \\
Workpiece weight(Kg/m) & 0.654 \\
Backend length & 8 \\
Allowance & 4 \\
Finish good length & 18 \\
\hline
\end{tabular}
propagation algorithm. This work uses supervised learning, with a set of training examples and the neural network via the learning algorithm implicitly rules. The topology of the proposed neural network model applies feed forward architecture. Each variable is the input value at a node of the input layer. The input layer of neuronal node is designed in such a way that one node is allocated for the feature type, and one node is allocated to each of the above sets of feature attributes.

Table 1 shows sample test input parameters, whereas the Table 2 shows the output parameters. There are three parts; actual data, prediction and errors. The ANN gives the best solution for extrusion process. It is clearly investigated that the actual yield is improved from 85 to $95 \%$ if the parameters are changed.

Figure 7 shows the training and learning process. There are 36 data are trained. The epoch is assigned by 1000 . The

\begin{tabular}{|c|c|c|c|c|c|c|c|c|}
\hline Actual & & & Prediction & & & $\%$ Error & & \\
\hline Billet length & $\begin{array}{l}\text { Finish good } \\
\text { qty }\end{array}$ & $\%$ Yield & Billet length & $\begin{array}{l}\text { Finish good } \\
\text { qty }\end{array}$ & $\%$ Yield & Billet length & $\begin{array}{l}\text { Finish good } \\
\text { qty }\end{array}$ & $\%$ Yield \\
\hline 13 & 5 & 85 & 14.11613 & 2.944283 & 99.31667 & 8.58 & 41.11 & 16.84 \\
\hline
\end{tabular}

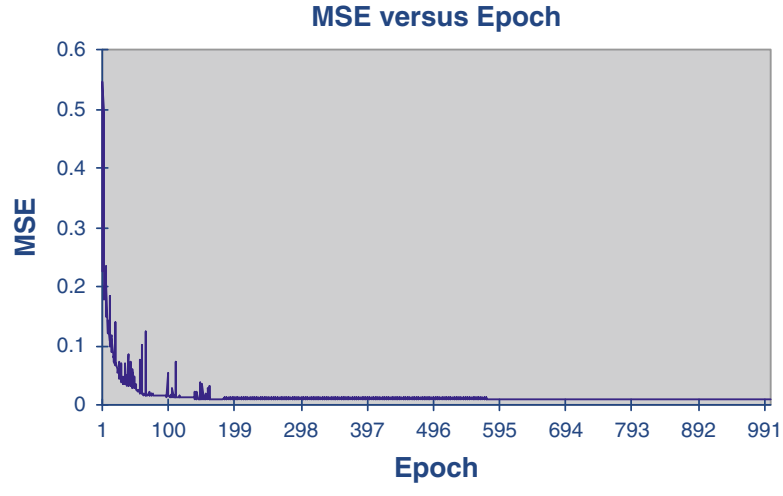

Training MSE 
best network is 616 epoch. The hidden layer is 6 layers. The error is 0.010586894 .

\section{Summary}

This chapter presents the methodology of case-based reasoning which is applied to adaptive aluminium extrusion die design on feature-based pattern matching. Instead of design from scratch, this approach search previous similar design problems and bring their solutions to solve current problems. However, the CBR is lack of dealing with numerical calculation and prediction. Therefore, this chapter is highlighted on applied the neural network to associate optimal extrusion processing parameters. It is clearly shown that the CBR is performed rapidly for feature die pattern matching together with the ANN can perform to give the best productivity of the yield.

\section{References}

1. Laue, K., Stenger, H. (1981) Extrusion Processes, Machinery, Tooling, 3rd ed. American Society for Metals, Metals Park, OH.

2. Negnevitsky, M. (2002) Artificial Intelligence: A Guide to Intelligent Systems. Person Addison Wesley, England
3. Singh, N. (1996) Systems Approach to Computer-Integrated Design and Manufacturing. Wiley, New York, NY.

4. Shah, J.J., Mentyla, M. (1995) Parametric and Feature-Based CAD/CAM, Concept, Techniques and Application. Wiley, New York, NY.

5. Thompson, W.B., et al. (1999) Feature-based reverse engineering of mechanical parts. IEEE Transactions on Robotics and Automation, 15:57-66.

6. Gayretli, A., Abdalla, H.S. (1999) A feature based prototypes system for the evaluation and optimization of manufacturing processes. Computer \& Industrial Engineering, 37:481-484.

7. Aamodt, A., Plaza, E. (1994) Case-based reasoning: Foundational issues methodological variations, and system approaches. AI Communications, 7:39-59.

8. Heylighen, A., Neuckermans, H. (2001) A case base of case-based design tools for architecture. Computer-Aided Design, 33:11111122.

9. Avramenko, Y., Kraslawski, A. (2006) Similarity concept for case-based design in process engineering. Computer \& Chemical Engineering, 30:548-557.

10. Vong, C.M., Leung, T.P., Wong, P.K. (2002) Case-based reasoning and adaptation in hydraulic production machine design. Engineering Application of Artificial Intelligence, 15: 567-585.

11. Tseng, M.M., Jiao, A. (1997) Case-based evolutionary design for mass customization. Computers and Industrial Engineering, 33(12):319-324.

12. Praszkiewicz, I.K. (2008) Application of artificial neural network for determination of standard time in machining. Journal of Intelligent Manufacturing, 19(2):233-240.

13. Scholz-Reiter, B., Hamann, T., Zschintzsch, M. (2007) Casebased reasoning for production control with neural networks. CIRP Journal of Manufacturing Systems, 36(1). 


\section{Chapter 50}

320

325

\section{AQ1 "S. Butdee" has been set as corresponding author. Please check. \\ AQ2 Please provide page number for reference [13].}

